\title{
Lost in Translation - \\ - the challenges with the use of animal models in translational research
}

Attila Seyhan ${ }^{1}$

${ }^{1}$ Fox Chase Cancer Institute, Temple Health, Temple University, Philadelphia, PA, USA

Key words: Preclinical and clinical research, animal models of human disease, reproducibility of research findings, translational medicine.

Correspondence:

Attila Seyhan, PhD, Attila_seyhan@yahoo.com; Mobile: 617-682-2954

The manuscript should be between $6-8$ pages in length (4,000 words). We encourage the use of illustrations, tables and figures to accompany the text; however, we may need to limit the number of color illustrations per contributor. 


\section{Contents}

$\begin{array}{ll}\text { Abstract } & 3\end{array}$

Introduction 4

$\begin{array}{ll}\text { Poor reproducibility of preclinical research } & 5\end{array}$

$\begin{array}{ll}\text { The limitations of using animals for human disease } & 8\end{array}$

$\begin{array}{ll}\text { Efforts to ameliorate animal research and reporting } & 11\end{array}$

Use of non-animal research methods $\quad 12$

$\begin{array}{ll}\text { The emerging model systems } & 14\end{array}$

$\begin{array}{ll}\text { Other approaches for improving translational efficiency } & 15\end{array}$

$\begin{array}{ll}\text { Summary } & 16\end{array}$

$\begin{array}{lr}\text { Conclusions } & 18\end{array}$

$\begin{array}{lr}\text { Review criteria } & 18\end{array}$

$\begin{array}{lr}\text { References } & 20\end{array}$ 


\section{Abstract}

Many preclinical research fails to replicate in human clinical trials; thus, the scale of poor translatability of preclinical research to human studies is widely recognized both in academia and industry. Potential reasons for this "Lost in Translation" problem could range from the complexity of modern biomedical research to the limitations of tools, the methodological differences, to poor experimental designs, inappropriate data analysis and misuse of statistics, and most importantly the reproducibility problems of animal results in humans. Poorly described protocols of research and low quality data presented, and inappropriate use of statistics all further magnify the problem.

Nonetheless, there are two major reasons for this: First, "Lost in Translation" problem, the "Butterfly Effect" (chaotic behavior of many preclinical animal models) and second, the "Two Cultures" problem (differences between the methodologies for preclinical and clinical research). Here we will focus on the animal models.

While the use of animals for scientific research is a traditional practice in biomedical research, it is also a matter of debate due to the animal welfare and ethical concerns of using animals for research. The apparent anatomical and physiological similarities amongst humans and animals, especially mammals, have made them ideal model systems to assess the efficacy and safety of novel therapies and mechanisms before testing them in humans. However, despite the interspecies similarities, many results resulting from animals do not directly translate to humans.

The purpose of this article is to conduct a thorough literature review to evaluate the nature of some of the problems resulting in poor translation of animal research to humans and to recommend some strategies to overcome this problem. 


\section{Introduction}

Ninety percent of the drugs entering human trials fail [1][2][3][4][5] and the process of getting a new drug to market is getting longer, costlier, and riskier business with high failure rates [6][7]. As discussed in this book and elsewhere [3], there are various reasons why drugs fail the development process.

One of the problems is the irreproducibility of many preclinical research findings and their poor translatability to human studies. There has been a growing argument that the failure of translation from preclinical findings to human outcomes may in part be due to the problems in the preclinical animal research itself $[10][11][12][13][14][15][16][17][18][19][20][21][22][23][24]$. On the other hand, some opinions argue that research on relevant, carefully designed, well-characterized and controlled animal models will remain a key step for important discoveries, for testing hypotheses at the organism level and for the testing of human data [25].

There could be many possible causes for the poor translatability for animal model research [26], in particular, the methodological flaws, such as underpowered studies, low group sizes, and lack of blinding [19] or whether appropriate animal models or species have been used.

Various recommendations to improve the translatability of animal model studies have been reported [27][28] including research on relevant, carefully designed, well-characterized and controlled animal models which will remain for a long time an essential step for fundamental discoveries, for testing hypotheses at the organism level and for the validation of human data.

However, due to long-standing issues with animal model studies, there is a renewed 
interest to focus on human studies for early discovery [22]. Recently, biopharmaceutical industry has begun disinvesting in internal animal research, which was taken up by academic and startup biotech sectors [11,29].

\section{Poor reproducibility of preclinical research}

Despite the enormous benefit of the preclinical research findings, there are many preclinical reports with irreproducible findings, dead ends in the search for new drugs for a number of human diseases. This is in part due to the significant differences between humans and animal such as genetic background, developmental span, and organ structure, and functions) make it challenging to translate findings in animals into a human disorders and corresponding therapies for human patients.

The research community has been well aware of the issues and merits involving translatability of various preclinical animal findings to human outcomes [2,3,5,24,38-44].

It has been estimated that just only six percent of animal studies translate to human response [45] and only about a third of highly cited animal research findings translates at the level of human trials $[19,46]$, and one-tenth of the interventions, are subsequently approved for use in patients.

The use of mouse and other animal models of human diseases have been the cornerstone of modern biomedical research $[47,48]$. The theory is that the findings from current mouse models developed to mimic human diseases translate directly to human conditions. However, this argument has been challenged by many studies including by Seok, et al. [36]. For example, researchers reported on the existence of 195 published methods that prevented or delayed the development of type 1 and 2 diabetes in mice [34], yet none of these "breakthroughs" ever translated to human medicine. 
In addition, mouse models of disease have been extensively used in modern biomedical research to identify and test the efficacy and safety of drug candidates for subsequent human studies [48,49]; however, translating animal studies to human studies have been poor and only a few of these human trials have been successful [50,51][52]. Particularly, the failure rate is even much greater for those trial in the area of inflammation, a condition present in many human diseases. As documented by Seok et al, approximately 150 clinical trials that tested candidate drug compounds intended to block the inflammatory response in patients failed [53][54][55][56].

In a recent article, researchers found that medical treatments developed in animals rarely translated to humans mostly due to poor replication of even high-quality animal studies [46]. Diseases that are artificially induced in animals in a laboratory, whether they be mice or monkeys, are never identical to those that occur naturally in human beings. Because animal species differ from one another biologically in many important ways, it becomes even more unlikely that animal experiments will generate results that will be correctly interpreted and translated to the human studies in a meaningful way.

Systematic studies evaluating how well animal models mimic human diseases are sparse. For example, Seok et al [36] have conducted a systematic analysis on the genomic responses of animal models and humans to similar inflammatory stresses and they found that, although acute inflammatory stresses from different etiologies result in highly similar genomic responses in humans, the responses in corresponding mouse models correlate poorly with the human conditions. As an example, among genes changed significantly in humans, the mouse ortholog genes were almost random in matching their human orthologs (e.g., R2 between 0.0 and 0.1 ).

Seok and colleagues [36] study supports a higher priority for translational medical research to focus on the more complex human conditions rather than relying on mouse models to study human inflammatory and other complex chronic diseases. They 
demonstrate that the complete genetic dissociation in their study challenges the usefulness of mice models in other areas of biomedical research and for drug development. In fact, many other researchers have been voicing their concerns about the shortcomings of the mice models of numerous other human diseases.

For example, according to former National Cancer Institute Director Dr. Richard Klausner, "We have cured mice of cancer for decades, and it simply didn't work in humans (Marlene Simmons et al., "Cancer-Cure Story Raises New Questions," Los Angeles Times 6 May 1998).

A recent opinion article [57] argues that even if the research were conducted rigorously, our ability to predict human responses from animal models will be limited by interspecies differences in molecular and metabolic pathways.

Similar concerns were highlighted in a widely cited paper, titled "Why most published research findings are false", that highlighted the problems caused by poor experimental and study design and analysis [39].

Another seminal paper published by a team at Bayer HealthCare reported that only about 25 percent of published preclinical studies could be validated [14]. Then, a paper published by Glenn Begley [13] has made this problem even more visible. Begley's test involved 53 studies that the results of these papers were purported to be groundbreaking. Most of the times the results could not be reproduced even by the original investigators themselves. Of the 53 original groundbreaking studies that Begley put to test, he could reproduce only six of them. A recent report published by Begley, titled, "Six red flags for suspect work". [13] brings forward the six most common but avoidable mistakes that he has identified. These included (1) whether experiments were performed blinded, (2) if basic experiments repeated, (3) whether all results presented or presented only the cherry-picked results; (4) whether the appropriate controls were 
used; (5) whether the reagents used in experiments were valid and high purity and quality; and (6) whether appropriate statistical tests and methods were employed.

His conclusion was that the appropriate scientific conduct was often not applied. Traditional training methods for biomedical research is often a haphazard process. Few basic scientists receive any formal teaching, most relying on what they learn from their supervisors and often imitate them [58] [59].

Despite the evidence that question the usefulness of animals to model human disease [48][60][47], in the absence of significant and successful translation of animal studies to human trials, many scientists and public regulators still assume that results from animal studies mirror human disease.

\section{The limitations of using animals for human disease}

What prevents most of the successes in animal experiments from becoming successful findings in human studies eventually? The reason is largely due to the species-specific differences.

Traditionally, many animal models are generated by inducing disease in otherwise healthy animals. Because of this, common comorbidity contexts of human patients in a specific disease may not be replicated in animal models. Animal models are limited in their capacity for self-reporting, therefore, the clinical endpoints must be carefully defined and reliable to distinguish treatment effects.

As discussed recently [61][14], contradictory findings have been found for every biological level of investigation-from genetics to physiology, from lifespan to metabolism, from biochemistry to immunology animals are different than humans. Furthermore, there are even deeper differences at the levels of nucleic acid, protein, pathway, cellular, tissue, organ, organism, and population levels, as well as their 
interaction with environment and response to environmental stimuli resulting in the difficulty of developing effective human treatments and the absence of cures.

Genetic and physiological variations within each species or between closely related species has led to the development of inbred strains which have highly homogeneous genetic composition to increase the reproducibility of results and the statistical power of experiments [25].

However, the disease clinical presentation and response to therapeutics can vary greatly between mouse strains. A recent report showed that while some mouse strains showed full resistance to Ebola virus, others died without specific symptoms whereas some other strains developed fatal hemorrhagic fever [62]. Differences in responses in animal strains might also underscore the differences in clinical observations among human patients.

The use of animal models of disease is not only the problem to blame for the high failure rate of biomedical research. It has been revealed recently [24] that many published research findings cannot be reproduced or not as robust as they were described. Begley has shown that only 11 percent of 53 seminal publications in preclinical cancer research could be confirmed by Amgen researchers [24,59].

The selection of the specific animal species for an animal model is crucial to study human disease. For example, a cholesterol-lowering drug failed in rats and one pharmaceutical company gave up on it entirely. But a Japanese researcher Arika Endo asked a colleague who was using chickens for experiments to test. The test results of this compound was a success in the birds which marked the emergence of statins, drugs used successfully to manage high cholesterol by millions of people.

However, nobody knows how well an animal model predicts a human. In fact test on mice may not even predict how a drug will work in another rodent. For example, certain 
drug-toxicity test run separately on rats and mice only resulted in for approximately 60 percent of the time.

Likewise, promising results in animal models often fail to translate to human. For example, mice with a mutation in the leptin gene that caused obesity in mice and treatment of leptin-deficient mice with leptin resulted in weight loss; however, studies in human with leptin supplements failed.

Furthermore, using animal models for research other than the commonly used models such as mice also presents a unique challenge for scientists such as financial feasibility and the usefulness of previous studies utilizing a given species for a specific human disease. Similarly, the unusual biological characteristics of a species and the available molecular techniques available for that particular species must also be considered. The choice of a naturally occurring species model must be identified which it can be most conveniently studied." One example is the use of the nine-banded armadillo in studies for leprosy due to the armadillo's unique susceptibility to M. leprae .

Since then, many studies have confirmed the failure to replicate published results including in areas ranging from physiology to cancer biology [63,64][65], diabetes [34][35][66][67][61], Alzheimer's [68], stroke [18], ALS [69][70,71], etc). The reasons of failure complex and multifaceted. As discussed in a recent popular book published by science journalist Richard Harris [59], they range from the complexity of biomedical science to the limitations of tools and training of scientists as well as incentives pressure to publish and secure funds.

Animal models often poorly predict results in human trials. Sample sizes could be too small to give reliable results not to mention the animal models are often too homogeneous and genetically identical. Not to mention other culprits ranging from the complexity of biology and biomedical science to the limitations of tools and training and contrary incentives in modern academia that wants to function businesses for profit. In 
the competitive field of science, several counterintuitive incentives conspire to undermine the scientific method, leading to a literature scattered with unreliable non-replicable data.

Animals still provide valuable information into fundamental biology if not the disease pathobiology itself. Nonetheless, translating that basic biology into medically relevant insights is complicated and that is even more complicated when cell lines or even primary human cells are used in research. Studies conducted in human cell lines have been shown to be problematic and prone to generate irreproducible data. It is not only the models that fail us but also biology itself. Evolution has created many redundant systems to ensure that organism is adaptive and survive and passes on their genes to next generation by adapting the dynamic environment rapidly. Many cancer drugs are good examples as well as diet drugs. Humans evolved many different biological signaling pathways and mechanisms to enable cellular growth and proliferation. Targeting one of those pathways is not going to block a specific disease phenotype entirely. This is why chemotherapy drugs eventually fail because often tumors eventually develop resistance to many chemotherapy drugs.

\section{Efforts to ameliorate animal research and reporting}

To address the significant shortcomings found in the conduct and reporting of preclinical research Sena et al., [72] proposed minimum quality standards for the range of animal research before findings from these studies are taken to human clinical trials.

Furthermore, to address this problem the ARRIVE (Animal Research: Reporting In Vivo Experiments) guidelines [73][57] were created. However, despite the endorsement of these guidelines by the major journals and funding agencies, the improvement in reporting standards was found to be marginal [74]. 
Systematic Review Centre for Laboratory Animal Experimentation (SYRCLE) in the Netherlands has created "A Gold Standard Publication Checklist" to promote more rigour in the conduct, not just reporting, of animal research [75].

It has also been acknowledged by $\mathrm{NIH}$ that poor training may in part be responsible for the lack of reproducibility of animal models [76]. There are resources such as workshops (www.3rs-reduction.co.uk) and training (www.syrcle.nl) are available for preclinical investigators in experimental design and statistical analysis, and an online course in experimental design and to learn how to conduct systematic reviews.

It has been reported that basic scientists' motivation comes from scientific discovery rather than the application of their findings to medicine $[58,77]$.

The "co-clinical trial" in which preclinical trials explicitly parallel ongoing human phase I and II trials have also been conducted [78] alongside with the development of translatibility scoring system to identify biomarkers that more accurately predict therapeutic outcomes [79].

\section{Use of non-animal research methods}

Recent developments in non-animal research methods have shown promise. For example, research conducted with human volunteers alongside with the aid of sophisticated computational methods, and in vitro studies based on human cells and tissues have been shown to be critically more effective and accurate than crude animal model studies.

There is also emerging new technologies such as organ-on-a-chip systems for animal-free toxicity testing [80][45,81-84][45,81-85]. These surrogate non-animal 
testing systems need not be perfect. It will be still highly impactful even if they are just significantly better than animal model systems and in vitro human studies.

As discussed in a recent commentary by Shuler, [45] there must be some advantages of organ-on-a-chip systems to have broad acceptance and impact, particularly on drug development. They need to be better than that of the existing animal model and human in vitro systems that are typically in the static momo-layer single type cell culture system. While animals present a fully integrated model, significant differences exist at the cellular, genetic and immune levels, as well as in size and pharmacokinetics and true biology and physiological response levels as well as pathophysiology and disease levels. These make translation of animal findings to human response difficult. Similarly, in vitro, human cell models often provide an incomplete assessment of complex fully integrated human response. Communication among cells within an organ or in between organs are critical for paracrine and endocrine signaling and to a response to a drug.

Development of drugs during preclinical research fails because both the insufficient efficacy and the toxicological side effects of the drug or its metabolites. New model systems that can identify in preclinical studies those drugs that will be both efficacious and have safety profile, could result in safer and more effective drugs at a relatively lower development cost not to mention saving the animal from unnecessary suffering.

There is no easy solution to this, however, by studying the disease using a combination of human cell cultures and tissues, in vitro and stem cell methods, and better understanding of disease mechanisms in patient samples, and other approaches directly relevant for patients may be a better alternative and more ethical and humane than that of animal models of disease studies. The NIH now has mandated that in grant proposals for applicants to specifically report how they will make sure that their results are robust and reproducible and many journals have now sections to ensure that the study has followed a list of criteria. In addition, there are new initiatives such as the one 
championed by Center for Open Science in Charlottesville, VA, which encourages open sharing of research process and resources including protocols, materials, and data. Furthermore, growing field of metascience - the scientific study of science may facilitate the progress in medical research and reduce the failure rate by analyzing large data sets.

\section{The emerging model systems}

Novel non-animal based models are becoming more available and have been shown to be more accurate than not so efficient animal experiments. Organs on a chip using induced pluripotent stem (iPS) cells taken from humans or in the future patients themselves is one promising model system. Those cells obtained from an individual with a particular disease can be induced in a miniaturized lab on a chip microfluidic device $[86,87]$ to become specific cell types or miniature organs with full physiological function not to mention genetically identical to the patient. This is a paradigm shift in early drug discovery and development that has been done traditionally in cell lines, human primary cell cultures, and in animal models of disease. The organ on a chip can also be used for drug safety and efficacy studies. However, the question remains how these organoids truly represent a fully functioning organ such as a liver or heart. Emulate, a spin-off of the Wyss Institute of Harvard has developed organoids using plastic chips built by 3D printers with intricate channels. These chips can be used to study organ biology and to screen and test candidate drugs for their efficacy. It is hoped that these model systems will demonstrate their value as a replacement of animal studies and that regulatory agencies will in near future accept them as a model system in place of animal studies.

It is inevitable that medical research that uses human volunteers, sophisticated computational methods, and advanced in vitro studies based on human organoids generated from iPS and tissues will be the future advancement of biomedical research [88][89]. 


\section{Other approaches for improving translational efficiency}

Small differences in preclinical studies including animal model designs can lead to large differences in effect size of therapeutics [90]. This is analogous to chaos theory, where small differences in initial conditions or model parameters may lead to large differences in results. Because of this, Ergorul and Levin [90] recommend applying strategies used by meteorologists in dealing with the chaos inherent in forecasting weather [91] to translational research. For example, in translational research, the characteristics of each model can be systematically varied in several dimensions. If the result remains the same despite changing parameters (e.g. the species, the method of inducing disease, the degree of injury, and other factors), it is more likely that the results will translate to human. These approaches are those of systems biology, and there are examples for their use in biology (e.g. translation initiation in eukaryotes [92] and differentiation of HL-60 cells [93]).

As discussed earlier [90], there are other methods for improving translational efficacy. For example, the phase 0 clinical trial is one where receptor binding, mechanism of action, pharmacodynamics, pharmacokinetics, or other biologic principles are studied in humans using microdoses of drug [94,95] without any therapeutic intent. The goal is to test whether the biological mechanism seen in animals can be affected by the treatment at a very low dose in a human. Phase 0 studies could be vital in improving the translational research process than at present. This is best done with a biomarker that could be highly sensitive to small concentrations of a drug tested.

Knowledge of a biological mechanism found in a disease can be employed to find new therapies, e.g. aberrant activation of Bcr-Abl tyrosine kinase in chronic myelocytic leukemia, which led to the development of the highly effective kinase inhibitor imatinib. Such bedside-to-bench observations in human disease can help focus the direction of 
animal research, which in turn will improve the translational process because they are already known to be associated with a clinical endpoint.

\section{Summary}

Data suggest that medical treatments developed in animals rarely translate to humans and; thus, researchers should remain cautious about extrapolating the finding of prominent animal research to the care of human disease. Moreover, irreproducibility of many animal studies is an additional concern on the value of this type of research. Likewise, diseases that are artificially induced in animals in a controlled laboratory environment are never identical to those that occur naturally in human beings. Because animal species differ from one another both in biology, physiology and in many other ways, it becomes even more unlikely that animal experiments will generate results that will be correctly interpreted and applied to the human condition in a meaningful way.

Further complicating the situation is the multifactorial nature of complex diseases such as cancer, cardiovascular diseases, infectious diseases, neurodegenerative disorders, pathological consequences of aging among others, it has been a challenge to develop efficacious therapies rapidly and efficiently.

To help overcome the challenges with the translational research, there needs to be some methodological changes in how experiments are designed and conducted, how animal models are used, and how preclinical and clinical teams work together to solve this "Lost in Translation" conundrum and increase the probability of preclinical findings that can be translated to the clinic.

Because of their complementarity, all experimental approaches such as genetics, genomics, biochemistry, physiology, in vitro and cell culture experiments, in silico modeling, animal models of disease, and clinical studies are indispensable. Therefore, preclinical research on carefully designed, well-characterized and controlled 
disease-relevant animal models will still remain a key stepping stone for testing early discoveries at the organism level and for the testing of human findings for the foreseeable future.

Other considerations include the applicability of the target, rigor, and validity of the experimental platform and models systems. Possible criteria may include greater than $50 \%$ increase in survival in case of animal models of cancer (three primary human orthopedic PDX models) and 50\% increase in survival of PDA GEMM. Additional considerations are route and schedule of delivery of agents similar to what will be used in humans. Demonstration of target inhibition, Duration of therapy that approximates anticipated duration in a trial. Matched data to the clinical indication and context such as stage of disease and the presence of intact immune response if appropriate (for immune therapeutics) and availability of biomarkers to predict responses.

Furthermore, the better use of the more complex human conditions and data rather than relying on mouse and other animal models to study human diseases. In addition, the use of non-animal techniques such as the use of human volunteers, epidemiological and clinical data sets, mathematical and computer (in silico) models, in vitro human tissue or organ models, and computer-modeling studies will improve overall biomedical research.

As importantly, "precision medicine" trials to evaluate targeted therapies combined with mechanism-based trials in which eligibility is based on criteria other than traditional disease definitions might help to improve overall biomedical research and the development of more efficacious therapies more efficiently in less time has been discussed in a recent report [96]. 


\section{Conclusions}

It is the author's opinion that future lies on the models that substitute animal models and these models must be constantly improved to go beyond animal models and mimic real human conditions. Likewise, biomedical research can be made more efficient by adopting new technologies and methods of scientific research and to better understand disease biology in humans.

\section{Review criteria}

Publicly available information such as PubMed and Internet were used for the literature review. We focused on identifying articles published on the translational research, use of animal models for human diseases, preclinical and clinical research, biomedical research, drug development, reproducibility of biomedical research data, failure of drug development and clinical trials. The search was restricted to the most recent studies in this field and all searches were limited to human studies published in English.

Figure 1. The amazing musicians of Bremen. Questionable grounds of animal research. 
The amazing musicians of Bremen.

Questionable grounds of animal research.

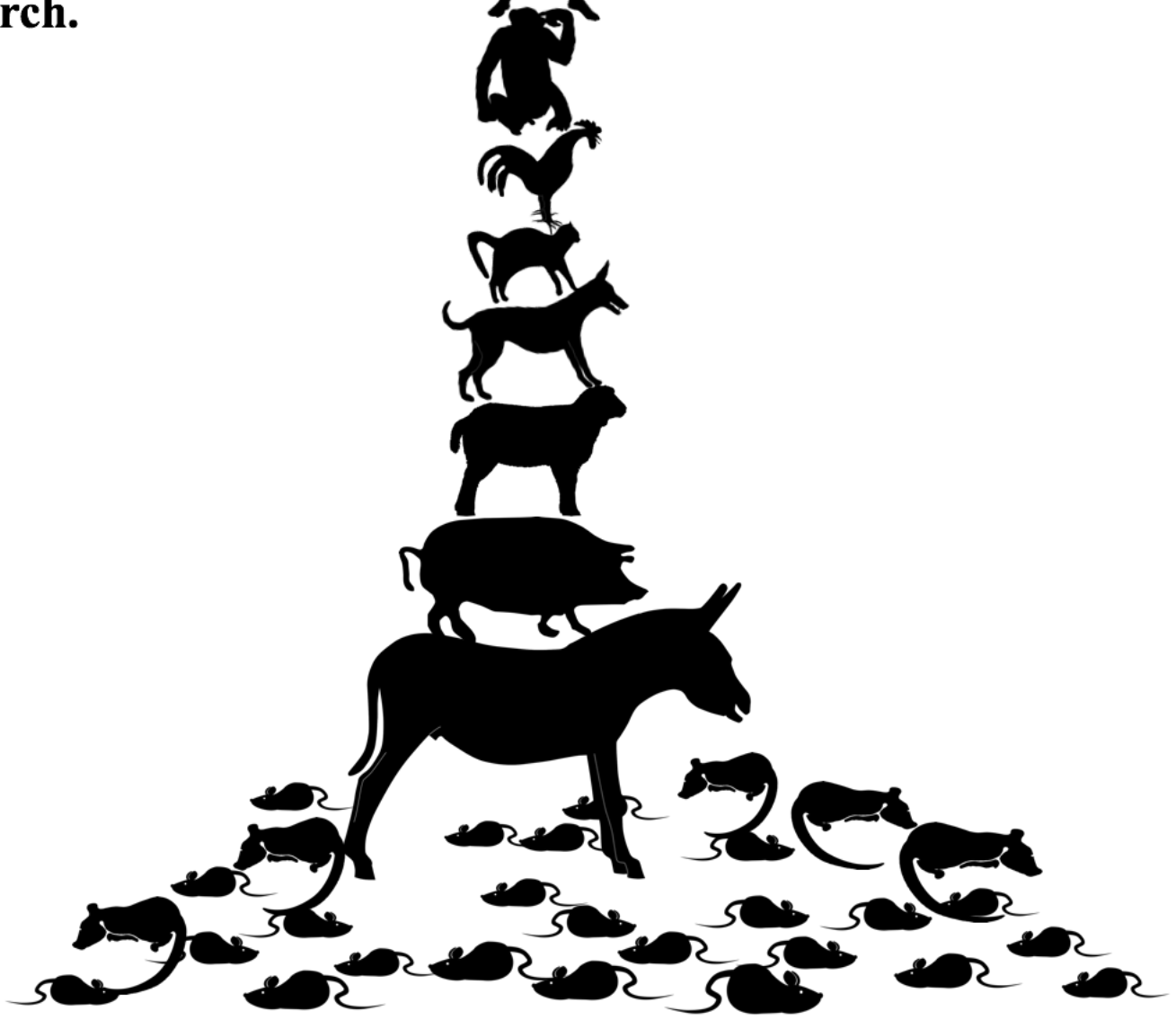




\section{References}

1. Garner JP, Gaskill BN, Weber EM, Ahloy-Dallaire J, Pritchett-Corning KR. Introducing Therioepistemology: the study of how knowledge is gained from animal research. Lab Anim . 2017;46: 103-113.

2. Paul SM, Mytelka DS, Dunwiddie CT, Persinger CC, Munos BH, Lindborg SR, et al. How to improve R\&D productivity: the pharmaceutical industry's grand challenge. Nat Rev Drug Discov. 2010; doi:10.1038/nrd3078

3. Kola I, Landis J. Can the pharmaceutical industry reduce attrition rates? Nat Rev Drug Discov. 2004;3: 711-715.

4. Scannell JW, Blanckley A, Boldon H, Warrington B. Diagnosing the decline in pharmaceutical R\&D efficiency. Nat Rev Drug Discov. 2012;11: 191-200.

5. Hay M, Thomas DW, Craighead JL, Economides C, Rosenthal J. Clinical development success rates for investigational drugs. Nat Biotechnol. 2014;32: 40-51.

6. DiMasi JA, Hansen RW, Grabowski HG. The price of innovation: new estimates of drug development costs. J Health Econ. 2003;22: 151-185.

7. Morgan S, Grootendorst P, Lexchin J, Cunningham C, Greyson D. The cost of drug development: A systematic review. Health Policy. 2011;100: 4-17.

8. Moher D, Shamseer L, Cobey KD, Lalu MM, Galipeau J, Avey MT, et al. Stop this waste of people, animals and money. Nature. 2017;549: 23-25.

9. Shen C, Björk B-C. "Predatory" open access: a longitudinal study of article volumes and market characteristics. BMC Med. 2015;13: 230.

10. Sabroe I, Dockrell DH, Vogel SN, Renshaw SA, Whyte MKB, Dower SK. Identifying and hurdling obstacles to translational research. Nat Rev Immunol. 2007;7: 77-82.

11. Rosenblatt M. An incentive-based approach for improving data reproducibility. Sci Transl Med. 2016;8: 336ed5.

12. Pusztai L, Hatzis C, Andre F. Reproducibility of research and preclinical validation: problems and solutions. Nat Rev Clin Oncol. 2013;10: 720-724.

13. Begley CG. Six red flags for suspect work. Nature. 2013;497: 433-434.

14. Prinz F, Schlange T, Asadullah K. Believe it or not: how much can we rely on published data on potential drug targets? Nat Rev Drug Discov. 2011;10: 712.

15. Mak IW, Evaniew N, Ghert M. Lost in translation: animal models and clinical trials in cancer treatment. Am J TransI Res. 2014;6: 114-118. 
16. Cummings JL, Morstorf T, Zhong K. Alzheimer's disease drug-development pipeline: few candidates, frequent failures. Alzheimers Res Ther. 2014;6: 37.

17. Zahs KR, Ashe KH. "Too much good news" - are Alzheimer mouse models trying to tell us how to prevent, not cure, Alzheimer's disease? Trends Neurosci. 2010;33: 381-389.

18. Sena ES, van der Worp HB, Bath PMW, Howells DW, Macleod MR. Publication bias in reports of animal stroke studies leads to major overstatement of efficacy. PLoS Biol. 2010;8: e1000344.

19. van der Worp HB, Howells DW, Sena ES, Porritt MJ, Rewell S, O'Collins V, et al. Can animal models of disease reliably inform human studies? PLoS Med. 2010;7: e1000245.

20. Peers IS, Ceuppens PR, Harbron C. In search of preclinical robustness. Nat Rev Drug Discov. 2012;11: 733-734.

21. Macleod MR, van der Worp HB, Sena ES, Howells DW, Dirnagl U, Donnan GA. Evidence for the efficacy of NXY-059 in experimental focal cerebral ischaemia is confounded by study quality. Stroke. 2008;39: 2824-2829.

22. Garner JP. The significance of meaning: why do over $90 \%$ of behavioral neuroscience results fail to translate to humans, and what can we do to fix it? ILAR J. 2014;55: 438-456.

23. Tricklebank MD, Garner JP. CHAPTER 20. The Possibilities and Limitations of Animal Models for Psychiatric Disorders. Drug Discovery. pp. 534-557.

24. Begley CG, Ellis LM. Drug development: Raise standards for preclinical cancer research. Nature. 2012;483: 531-533.

25. Barré-Sinoussi $F$, Montagutelli $X$. Animal models are essential to biological research: issues and perspectives. Future Sci OA. 2015;1: FSO63.

26. Sabroe I, Dockrell DH, Vogel SN, Renshaw SA, Whyte MKB, Dower SK. Identifying and hurdling obstacles to translational research. Nat Rev Immunol. 2007;7: 77-82.

27. Ioannidis JPA. Clinical trials: what a waste. BMJ. 2014;349: g7089-g7089.

28. Ioannidis JPA, Greenland S, Hlatky MA, Khoury MJ, Macleod MR, Moher D, et al. Increasing value and reducing waste in research design, conduct, and analysis. Lancet. 2014;383: 166-175.

29. Hunter J. Challenges for pharmaceutical industry: new partnerships for sustainable human health. Philos Trans A Math Phys Eng Sci. 2011;369: 1817-1825.

30. Michael Conn P. Animal Models for the Study of Human Disease. Academic Press; 2017.

31. Tannenbaum J. Animal Models for the Study of Human Disease: Chapter 1. Ethics in Biomedical Animal Research: The Key Role of the Investigator. Elsevier Inc. Chapters; 
2013.

32. Newkirk I. The PETA Practical Guide to Animal Rights: Simple Acts of Kindness to Help Animals in Trouble. St. Martin's Griffin; 2009.

33. Holen I, Speirs V, Morrissey B, Blyth K. In vivo models in breast cancer research: progress, challenges and future directions. Dis Model Mech. 2017;10: 359-371.

34. Roep BO, Atkinson M, von Herrath M. Opinion: Satisfaction (not) guaranteed: re-evaluating the use of animal models of type 1 diabetes. Nat Rev Immunol. 2004;4: 989-997.

35. Chen Y-G, Mathews CE, Driver JP. The Role of NOD Mice in Type 1 Diabetes Research: Lessons from the Past and Recommendations for the Future. Front Endocrinol . 2018;9. doi:10.3389/fendo.2018.00051

36. Seok J, Warren HS, Cuenca AG, Mindrinos MN, Baker HV, Xu W, et al. Genomic responses in mouse models poorly mimic human inflammatory diseases. Proc Natl Acad Sci U S A. 2013;110: 3507-3512.

37. Cavanaugh SE. Animal models of Alzheimer disease: historical pitfalls and a path forward. ALTEX. 2014;31: 279-302.

38. Geerts $\mathrm{H}$. Of mice and men: bridging the translational disconnect in CNS drug discovery. CNS Drugs. 2009;23: 915-926.

39. Ioannidis JPA. Why Most Published Research Findings Are False. Chance . 2005;18: 40-47.

40. Viktor R. Environmental Enrichment and Refinement of Handling Procedures. The Laboratory Primate. 2005. pp. 209-227.

41. Richter SH, Garner JP, Würbel H. Environmental standardization: cure or cause of poor reproducibility in animal experiments? Nat Methods. 2009;6: 257-261.

42. Richter SH, Helene Richter S, Garner JP, Auer C, Kunert J, Würbel H. Systematic variation improves reproducibility of animal experiments. Nat Methods. 2010;7: 167-168.

43. Insel TR. From Animal Models to Model Animals. Biol Psychiatry. 2007;62: 1337-1339.

44. Gaskill BN, Garner JP. Stressed out: providing laboratory animals with behavioral control to reduce the physiological effects of stress. Lab Anim . 2017;46: 142-145.

45. Shuler ML. Organ-, body- and disease-on-a-chip systems. Lab Chip. 2017;17: 2345-2346.

46. Hackam DG, Redelmeier DA. Translation of research evidence from animals to humans. JAMA. 2006;296: 1731-1732.

47. Hayday AC, Peakman M. The habitual, diverse and surmountable obstacles to human 
immunology research. Nat Immunol. 2008;9: 575-580.

48. Woodcock J, Woosley R. The FDA critical path initiative and its influence on new drug development. Annu Rev Med. 2008;59: 1-12.

49. Vargas HM, Amouzadeh HR, Engwall MJ. Nonclinical strategy considerations for safety pharmacology: evaluation of biopharmaceuticals. Expert Opin Drug Saf. 2012;12: 91-102.

50. Does animal research benefit humans? BMJ. 2004;328: 0-e-0.

51. Pound P. Where is the evidence that animal research benefits humans? BMJ. 2004;328: 514-517.

52. Rice J. Animal models: Not close enough. Nature. 2012;484: S9-S9.

53. Hotchkiss RS, Opal S. Immunotherapy for sepsis--a new approach against an ancient foe. N Engl J Med. 2010;363: 87-89.

54. Hotchkiss RS, Coopersmith CM, McDunn JE, Ferguson TA. The sepsis seesaw: tilting toward immunosuppression. Nat Med. 2009;15: 496-497.

55. Wiersinga WJ. Current insights in sepsis: from pathogenesis to new treatment targets. Curr Opin Crit Care. 2011;17: 480-486.

56. Mitka M. Drug for severe sepsis is withdrawn from market, fails to reduce mortality. JAMA. 2011;306: 2439-2440.

57. Pound $P$, Bracken $M B$. Is animal research sufficiently evidence based to be a cornerstone of biomedical research? BMJ. 2014;348: g3387.

58. Festing MFW, Nevalainen T. The Design and Statistical Analysis of Animal Experiments: Introduction to this Issue. ILAR J. 2014;55: 379-382.

59. Harris R. Rigor Mortis: How Sloppy Science Creates Worthless Cures, Crushes Hope, and Wastes Billions. Hachette UK; 2017.

60. Davis MM. A Prescription for Human Immunology. Immunity. 2008;29: 835-838.

61. Chandrasekera PC, Pippin JJ. Of rodents and men: species-specific glucose regulation and type 2 diabetes research. ALTEX. 2014;31: 157-176.

62. Rasmussen AL, Okumura A, Ferris MT, Green R, Feldmann F, Kelly SM, et al. Host genetic diversity enables Ebola hemorrhagic fever pathogenesis and resistance. Science. 2014;346: 987-991.

63. Day C-P, Merlino G, Van Dyke T. Preclinical mouse cancer models: a maze of opportunities and challenges. Cell. 2015;163: 39-53.

64. Gengenbacher N, Singhal M, Augustin HG. Preclinical mouse solid tumour models: status 
quo, challenges and perspectives. Nat Rev Cancer. 2017;17: 751-765.

65. Zhao X, Li L, Starr TK, Subramanian S. Tumor location impacts immune response in mouse models of colon cancer. Oncotarget. 2017;8: 54775-54787.

66. DIAbetes Genetics Replication And Meta-analysis (DIAGRAM) Consortium, Asian Genetic Epidemiology Network Type 2 Diabetes (AGEN-T2D) Consortium, South Asian Type 2 Diabetes (SAT2D) Consortium, Mexican American Type 2 Diabetes (MAT2D) Consortium, Type 2 Diabetes Genetic Exploration by Nex-generation sequencing in muylti-Ethnic Samples (T2D-GENES) Consortium, Mahajan A, et al. Genome-wide trans-ancestry meta-analysis provides insight into the genetic architecture of type 2 diabetes susceptibility. Nat Genet. 2014;46: 234-244.

67. Dupuis J, Langenberg C, Prokopenko I, Saxena R, Soranzo N, Jackson AU, et al. New genetic loci implicated in fasting glucose homeostasis and their impact on type 2 diabetes risk. Nat Genet. 2010;42: 105-116.

68. Cavanaugh SE. Animal models of Alzheimer disease: historical pitfalls and a path forward. ALTEX. 2014;31: 279-302.

69. Rothstein JD. Preclinical studies: how much can we rely on? Amyotroph Lateral Scler Other Motor Neuron Disord. 2004;5 Suppl 1: 22-25.

70. Traynor BJ, Bruijn L, Conwit R, Beal F, O’Neill G, Fagan SC, et al. Neuroprotective agents for clinical trials in ALS: A systematic assessment. Neurology. 2006;67: 20-27.

71. Benatar M. Lost in translation: Treatment trials in the SOD1 mouse and in human ALS. Neurobiol Dis. 2007;26: 1-13.

72. Sena E, van der Worp HB, Howells D, Macleod M. How can we improve the pre-clinical development of drugs for stroke? Trends Neurosci. 2007;30: 433-439.

73. Kilkenny C, Browne WJ, Cuthill IC, Emerson M, Altman DG. Improving bioscience research reporting: the ARRIVE guidelines for reporting animal research. PLoS Biol. 2010;8: e1000412.

74. Baker D, Lidster K, Sottomayor A, Amor S. Two Years Later: Journals Are Not Yet Enforcing the ARRIVE Guidelines on Reporting Standards for Pre-Clinical Animal Studies. PLoS Biol. 2014;12: e1001756.

75. Hooijmans CR, Leenaars M, Ritskes-Hoitinga M. A gold standard publication checklist to improve the quality of animal studies, to fully integrate the Three Rs, and to make systematic reviews more feasible. Altern Lab Anim. 2010;38: 167-182.

76. Collins FS, Tabak LA. Policy: NIH plans to enhance reproducibility. Nature. 2014;505: 612-613.

77. Morgan M, Barry CA, Donovan JL, Sandall J, Wolfe CDA, Boaz A. Implementing 
"translational" biomedical research: Convergence and divergence among clinical and basic scientists. Soc Sci Med. 2011;73: 945-952.

78. Chen Z, Cheng K, Walton Z, Wang Y, Ebi H, Shimamura T, et al. A murine lung cancer co-clinical trial identifies genetic modifiers of therapeutic response. Nature. 2012;483: 613-617.

79. Wendler A, Wehling M. Translatability scoring in drug development: eight case studies. J TransI Med. 2012;10: 39.

80. Mahler GJ, Esch MB, Stokol T, Hickman JJ, Shuler ML. Body-on-a-chip systems for animal-free toxicity testing. Altern Lab Anim. 2016;44: 469-478.

81. Bessems J. Toxicokinetic modelling: A necessary tool for quantitative risk assessment in animal-free toxicity testing. Toxicol Lett. 2015;238: S47.

82. Leist M. Consensus report on the future of animal-free systemic toxicity testing. ALTEX. 2008; 341-356.

83. Kessler R. Filling a Gap in Developmental Toxicity Testing: Neural Crest Cells Offer Faster, Cheaper, Animal-Free Testing. Environ Health Perspect. 2012;120: a320-a320.

84. Materne E-M, Tonevitsky AG, Marx U. Chip-based liver equivalents for toxicity testing organotypicalness versus cost-efficient high throughput. Lab Chip. 2013;13: 3481.

85. Oleaga C, Bernabini C, Smith AST, Srinivasan B, Jackson M, McLamb W, et al. Multi-Organ toxicity demonstration in a functional human in vitro system composed of four organs. Sci Rep. 2016;6. doi:10.1038/srep20030

86. Ozcan A. Mobile phones democratize and cultivate next-generation imaging, diagnostics and measurement tools. Lab Chip. 2014;14: 3187-3194.

87. Ozcan A. Democratization of Next-Generation Imaging, Diagnostics and Measurement Tools through Computational Photonics. Biophys J. 2015;108: 371a.

88. Bredenoord AL, Clevers $\mathrm{H}$, Knoblich JA. Human tissues in a dish: The research and ethical implications of organoid technology. Science. 2017;355: eaaf9414.

89. Xinaris C, Brizi V, Remuzzi G. Organoid Models and Applications in Biomedical Research. Nephron. 2015;130: 191-199.

90. Ergorul C, Levin LA. Solving the lost in translation problem: improving the effectiveness of translational research. Curr Opin Pharmacol. 2013;13: 108-114.

91. Shukla J. Predictability in the Midst of Chaos: A Scientific Basis for Climate Forecasting. Science. 1998;282: 728-731.

92. Nayak S, Siddiqui JK, Varner JD. Modelling and analysis of an ensemble of eukaryotic 
translation initiation models. IET Syst Biol. 2011;5: 2.

93. Tasseff R, Nayak S, Song SO, Yen A, Varner JD. Modeling and analysis of retinoic acid induced differentiation of uncommitted precursor cells. Integr Biol . 2011;3: 578-591.

94. Kummar S, Rubinstein L, Kinders R, Parchment RE, Gutierrez ME, Murgo AJ, et al. Phase 0 Clinical Trials: Conceptions and Misconceptions. Cancer J Sci Am. 2008;14: 133-137.

95. LoRusso PM. Phase 0 Clinical Trials: An Answer to Drug Development Stagnation? J Clin Oncol. 2009;27: 2586-2588.

96. Woodcock J, LaVange LM. Master Protocols to Study Multiple Therapies, Multiple Diseases, or Both. N Engl J Med. 2017;377: 62-70. 